\title{
Shear Viscosity of Benzene, Toluene, and $p$-Xylene by Non-equilibrium Molecular Dynamics Simulations
}

\author{
Song Hi Lee \\ Department of Chemistrv, Kumgsung Chiversin, Busan 608-736, Korea \\ Received November 26, 2003
}

Key Words : Shear viscosity. Molecular dỵnamics simulation. Benzene. Toluene. $p$-Xylene

Green and Kubo ${ }^{1}$ showed that the phenomenological coefficients describing many transport processes and timedependent phenomena in general could be written as integrals over a certain type of function called a timecorrelation function. The Green-Kubo formulas are the formal expressions for hydrodynamic field variables and some of the thermodynamic properties in terms of the microscopic variables of an $\mathrm{N}$-particle system. The identification of microscopic expressions for macroscopic variables is made by a process of comparison of the conservation equations of hydrodynamics with the microscopic equations of change for conserved densities. The importance of these formulas is three-fold: they provide an obvious method for calculating transport coefficients using computer simulation. a convenient starting point for constructing analy tic theories for non-equilibrium processes, and an essential information for designing non-equilibrium molecular dynanics (NEMD) algorithm. The Green-Kubo formula for the shear viscosity is given by

$$
\eta=\frac{V}{k T} \int_{0}^{\infty} \mathrm{dt}\left\langle P_{\alpha \beta}(0) \cdot P_{\alpha \beta}(t)\right\rangle
$$

where $P_{\alpha \beta \beta}$ is an off-diagonal $(\alpha \neq \beta)$ of the viscous pressure tensor:

$$
P_{\alpha \beta}(t)=\frac{1}{V}\left[\sum_{s} m v_{r \alpha}(t) \cdot v_{s \beta}(t)+\sum_{i} r_{r \alpha}(t) \cdot r_{s \beta}(t)\right] .
$$

In recent years, non-equilibrium molecular dynamics (NEMD) sinulation has emerged as a powerful tool for the study of transport coefficients of both simple and molecular fluids. The general principle of the NEMD method ${ }^{2}$ is to introduce a (possibly fictitious) external field $\mathrm{X}$ into the equations of motion of the system. which derives the corresponding themodynamic flux $J$. The first requirement for this applied field is that it should be consistent with the periodic boundary conditions to ensure that the simulation box remains homogeneous. The second requirement is that the transport coefficient $\gamma$ of interest can be calculated from the constitutive relation:

$$
\gamma=\lim _{X \rightarrow 0}\left[\lim _{t \rightarrow \infty} \frac{\langle J(t)\rangle}{X}\right] .
$$

The formal proof that an algorithm satisfies these two requirements is given by linear response theory. ${ }^{3.5}$

Among many NEMD methods developed for calculating the shear viscosity the most efficient technique appears to be the Sllod algorithm ${ }^{2.3}$ a standard method using homogeneous Lees-Edwards "sliding brick" boundary conditions. ${ }^{.}$This algorithm sets up a steady state planar Couette flow with the two plates moving in opposite $x$ directions located at $y= \pm \infty$ so that the streaming velocity has a non-zero component in the $x$ direction $\mathrm{du}_{\mathrm{l}} / \mathrm{dy}=\gamma_{\text {where }} \gamma_{\text {is }}$ the constant strain rate.

Following Evans and Morriss., ${ }^{2,3}$ the equations of translational motion for the center of mass in a molecular fluid are given by:

$$
\begin{gathered}
\dot{\boldsymbol{r}}_{i}=\boldsymbol{p}_{i} / m_{i}+\boldsymbol{r}_{i} \cdot \nabla \boldsymbol{u} \\
\dot{p}_{i}=\boldsymbol{F}_{i}-\alpha_{i} p_{i}-p_{i} \cdot \nabla \boldsymbol{u}
\end{gathered}
$$

where $u=\left(\begin{array}{lll}u_{x} & 0 & 0\end{array}\right)$ with $u_{x}=\gamma y$ is the velocity field corresponding to planar Couette flow.

The equations of rotational motion about the center of mass for molecular fluids are derived using quaternions ${ }^{7.8}$ follows:

$$
\begin{aligned}
& \dot{L}_{i}=T_{l}-\alpha_{r} L_{2} \\
& L_{j}^{p}=A_{i} L_{i} \\
& \omega_{i k}^{p}=\frac{L_{k \dot{k}}}{I_{k}}, \quad k=x, y, z \\
& \left(\begin{array}{l}
\dot{q}_{i 1} \\
\dot{q}_{i 2} \\
\dot{q}_{t 3} \\
\dot{q}_{i+1}
\end{array}\right)=\frac{1}{2}\left(\begin{array}{cccc}
-q_{i 3} & -q_{i 1} & q_{i 2} & q_{i 1} \\
q_{i 1} & -q_{i 3} & -q_{i 1} & q_{i 2} \\
q_{11} & q_{r 2} & q_{t 3} & q_{s 3} \\
-q_{t 2} & q_{i 1} & -q_{t 3} & q_{i 4}
\end{array}\right)\left(\begin{array}{c}
\omega_{i x}^{p} \\
\omega_{i v}^{p} \\
\omega_{s=}^{p} \\
0
\end{array}\right) .
\end{aligned}
$$

where $L_{i}$ is the angular momentum of molecule $i_{i} T_{i}$ is the torque on molecule $i$ in the laboratory frame. $L_{s}^{p}$ and $\omega_{t}^{\prime}$, are, respectively. the angular momentum and angular velocities of molecule $i$ in its principle axis frame, $A_{i}$ is the rotation matrix which transforms vectors form the laboratory to principal axis frame of molecule $i . I_{k}$ is the principal axis of momenta of inertia of each molecule, and $q_{l k}, k=1,2.3 .4$, are the quaternion parameters related to the Euler angles describing the orientation of molecule $i$ in space. The translational and rotational temperature constraint parameters $\alpha_{t}$ and $\alpha_{r}$ are given by

$$
\alpha_{i}=\sum_{i}^{*}\left[p_{i} \cdot F_{i}\right] / \sum_{i}^{N} p_{i}^{2}
$$


and

$$
\alpha_{r}=\sum_{i}^{N}\left[L_{i} \cdot T_{i}\right] / \sum_{j}^{N} L_{i}^{2} .
$$

These equations of motion are combined with the LeesEdwards "sliding brick" boundary conditions." In the absence of the thermostat and the isobaric constraint the terms in Eqs. (4) and (5) involving the strain field. $\gamma$. cancel to yield Newton's equations of motion relating $\boldsymbol{r}_{3}$, and $\boldsymbol{F}_{3}$. This implies that the Sllod algorithm truly generates boundary driven planar Couette flow, leading to the conclusion that it is correct to arbitrary order in the strain rate. ${ }^{3}$ In order to obtain a good signal-to-noise ratio. with NEMD it is necessary to use strain rates $\gamma$ which are high enough to cause the shear viscosity to be strain rate dependent. In order to compute the shear viscosity of a Newtonian fluid using the Sllod algorithm, after the simulation reaches the steady state at a given strain rate $\gamma$ one computes and averages the pressure tensor defined in Eq. (2). The strain rate dependent shear viscosity is then obtained from Newton's law of viscosity

$$
\eta=\frac{P_{x s}+P_{s i x}}{2 \gamma}
$$

where $P_{x y^{\prime}}$ and $P_{y x}$ are the averaged $\mathrm{xy}$ and $\mathrm{yx}$ components of $P$. From kinetic and mode coupling theories it is known ${ }^{5-1 l}$ that to leading order the strain rate dependence of the shear viscosity is linear in $\gamma^{1}$. Hence. to apply the Sllod algorithm to a Newtonian fluid. one performs several simulations at differing strain rates $\gamma$ and fits the resulting strain dependent viscosities to the equation:

$$
\eta=\eta_{0}+\eta_{1} \gamma^{2}
$$

The zero strain rate extrapolation of $\eta$. $\eta_{0 .}$ is thus the Newtonian viscosity.

The molecular model used is the same as in the previous MD simulations ${ }^{12.13}$ : the benzene ring is assumed as a rigidbody. but three $\mathrm{C}-\mathrm{H}$ bonds in the methyl groups of toluene $\left(\mathrm{C}_{6} \mathrm{H}_{5} \mathrm{CH}_{3}\right)$ and $p-x y$ lene $\left(\mathrm{CH}_{3} \mathrm{C}_{6} \mathrm{H}_{4} \mathrm{CH}_{3}\right)$ are not assumed to be rigid. Simple hamonic oscillation potentials for $\mathrm{C}-\mathrm{H}$ bond stretching and $\mathrm{C} \cdot \mathrm{C} \cdot \mathrm{H}$ and $\mathrm{H}-\mathrm{C} \cdot \mathrm{H}$ bond angle bending in the methyl group are used. and the methyl groups are rotating according to a torsional potential. Potential parameters for the molecular model are given in Ref. 12. The usual periodic boundary condition in the $x-y-$, and $z-$ directions and minimum inage convention for pair potential were applied. A spherical cut-off of radius $R_{c}=12.5 \AA$ was employed for the pair interactions. For the integration over time, we adopted Gear's fifth order predictor-corrector algorithm $^{14}$ with a time step of $0.005 \mathrm{ps}$ for benzene and $0.0005 \mathrm{ps}$ for toluene and $p$-sylene. All NEMD simulations in canonical (NVT) ensembles were carried on 120 molecules and fully equilibrated for about several $1.000,000$ time steps for each liquid molecular system at $293.15 \mathrm{~K}$. The equilibrium properties were then averaged over 5 blocks of $2.000,000$ time steps for a total of $10,000.000$ time steps. and the configurations of molecules were stored every 10 time steps for analyses of structural and dynamic properties.

\section{Results and Discussion}

The results are summarized in Table $\mathrm{l}$ for the liquid benzene, toluene, and p-xylene at $293.15 \mathrm{~K}$. The pressure, LJ energy, and shear viscosity are reported as a function of strain rate $(\gamma)$. Figure 1 shows the shear viscosity as a function of $\gamma^{\text {la }}$ for these systems. The calculated shear viscosities of these sy stems at lower strain rates for $\gamma^{1 / 2} \leq 0.6$ $\mathrm{ps}^{1: 2}$ fit the theoretical prediction. Eq. (13), quite well, but those at higher strain rates for $\gamma^{12} \geq 0.7 \mathrm{ps}^{1}$ are not a linear function of $\gamma$. Hence the zero strain rate extrapolation of the shear viscosities at $\gamma^{1: 2}=0.1-0.6 \mathrm{ps}^{1: 2}$. shown as least-squares fitted straight lines in Figure 1 , is used to determine the values of the shear viscosity which are listed in Table $\mathrm{I}$ at $\gamma=$ 0.0 . At the highest strain rate. $\gamma=1.0 \mathrm{ps}$, the magnitude of calculated shear viscosities is in the order of $p$-xylene. toluene, and benzene. As the strain rate decreases. the calculated shear viscosity of benzene becomes bigger than

\begin{tabular}{|c|c|c|c|c|c|c|c|c|c|}
\hline \multirow[b]{2}{*}{$\gamma$} & \multicolumn{3}{|c|}{ benzene $(0.928)^{a}$} & \multicolumn{3}{|c|}{ toluene $(0.933)^{4}$} & \multicolumn{3}{|c|}{$p$-xylene $(0.953)^{a}$} \\
\hline & $\mathrm{p}$ & $\mathrm{E}_{\mathrm{LN}}$ & $\eta$ & $\mathrm{p}$ & $E_{L .1}$ & $\eta$ & $\mathrm{p}$ & $\mathrm{E}_{\mathrm{L}, \mathrm{I}}$ & $\eta$ \\
\hline 1.00 & 861 & -28.44 & 0.198 & -590 & -32.81 & 0.210 & -70 & -37.58 & 0.236 \\
\hline 0.81 & 528 & -28.69 & 0.219 & -831 & -33.05 & 0.231 & -352 & -37.94 & 0.257 \\
\hline 0.64 & 207 & -28.84 & 0.246 & -1073 & -33.22 & 0.255 & -622 & -38.19 & 0.283 \\
\hline 0.49 & -100 & -28.93 & 0.278 & -1284 & -33.33 & 0.286 & -868 & -38.37 & 0.314 \\
\hline 0.36 & -381 & -28.96 & 0.323 & -1473 & -33.39 & 0.323 & -1083 & -38.48 & 0.354 \\
\hline 0.25 & -627 & -29.01 & 0.382 & -1627 & -33.44 & 0.375 & -1271 & -38.56 & 0.403 \\
\hline 0.16 & -836 & -29.11 & 0.448 & -1751 & -33.51 & 0.435 & -1417 & -38.61 & 0.462 \\
\hline 0.09 & -994 & -29.20 & 0.524 & -1846 & -33.55 & 0.505 & -1530 & -38.64 & 0.525 \\
\hline 0.04 & -1104 & -29.26 & 0.607 & -1903 & -33.57 & 0.581 & -1601 & -38.64 & 0.599 \\
\hline 0.01 & -1160 & -29.28 & 0.670 & -1928 & -33.65 & 0.637 & -1625 & -38.75 & 0.652 \\
\hline 0.0 & -1200 & -29.28 & $0.741^{b}$ & -1940 & -33.60 & $0.702^{b}$ & -1633 & -38.64 & $0.713^{b}$ \\
\hline
\end{tabular}

Table 1. The pressure ( $\mathrm{p}$ in atm), LJ energy ( $\mathrm{E}_{\mathrm{LJ}}$ in $\mathrm{k} . \mathrm{T} / \mathrm{mol}$ ), and viscosity ( $\eta$ in $\mathrm{cP}$ ) of the liquid benzene, toluene, and $p$ - $\mathrm{xylene}$ at $293.15 \mathrm{~K}$ as functions of strain rate $\left(y /\right.$ in $\left.\mathrm{ps}^{-1}\right)$

"Density $\left(\mathrm{g} \mathrm{cm}^{3}\right)$ of each simulated system. "The extrapolated value. 


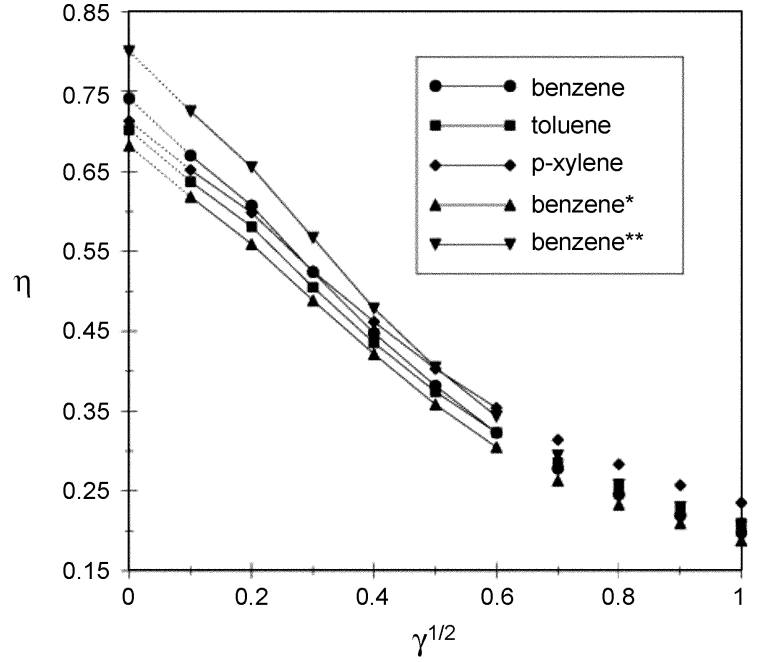

Figure 1. Shear viscosities (in units of eP) for liquid benzene. toluene. and $x-x y$ lene at 293.15 . Benzene" and benzene" represent the results at the density of 0.917 and $0.939 \mathrm{~g} / \mathrm{cm}^{3}$. respectively.

that of toluene at $\gamma<0.36 \mathrm{ps}$ and bigger than that of $p$-xylene at $\gamma<0.09 \mathrm{ps}$.

The calculated viscosities of the liquid benzene, toluene, and $p$-xylene from our NEMD simulations are slightly higher than the experimental results of the viscosity for these systems at $293.15 \mathrm{~K}$ which are 0.652 .0 .590 , and $0.648 \mathrm{cP}^{15}$ but those are generally in good agreement. The current choice of the density of each simulated system is rather fortunate. In the previous study of the isobaric-isothermal (Npl) MD simulations for these systems. ${ }^{1:}$ the determined density of each system is given in Table 2 . These values of density were apparently too high when compared with the experimental ones $-0.87865,0.8669$, and $0.8611 \mathrm{~g} / \mathrm{cm}^{3}$, respectively. The previous canonical (NVI) MD simulations study for the same systems ${ }^{12}$ of the experimental densities gave very low pressures as shown in Table 2. which invoked restudy of these systems by NpT MD simulations. ${ }^{1.3}$ This

Table 2. Irensity $\left(\mathrm{g} / \mathrm{cm}^{3}\right)$ and pressure (atm) of the liquid bencene. toluene. and $p$-xy/ene at $293.15 \mathrm{~K}$ by experiment and molecular dinamics (MD) simulations

\begin{tabular}{lccc}
\hline & benzene & toluene & $p$-xylene \\
\hline density by expariment & 0.87865 & 0.8669 & 0.8611 \\
pressure by NVT MD ${ }^{27}$ & -1160 & -1670 & -1840 \\
density by NPT MD & 0.985 & 1.040 & 0.993 \\
density in this work & 0.928 & 0.933 & 0.953 \\
\hline
\end{tabular}

"Ref. I2. The simulation density is the one by experiment. "Ref. 13. should be pointed out as a possible limitation of this model with regard to the system density.

The calculated viscosities of these systems are very sensitive to the choice of the density. For example, Fig. I compares three sets of shear viscosities of benzene at three different densities of 0.917 (benzene"). 0.928(benzene), and 0.939 (benzene $^{* *}$ ) $\mathrm{g} / \mathrm{cm}^{3}$ which are corresponding to the lengths of the simulation boxes of 2.57, 2.56, and $2.55 \mathrm{~nm}$. respectively. The resulting viscosities at these densities are $0.682,0.741$, and $0.80 \mathrm{l} \mathrm{cP}$, respectively. The first value of the viscosity is closest to the experimental one, but taking account of the order of the viscosity for liquid benzen. toluene, and $p$-xylene, the second one is most reasonable value.

We also calculated the shear viscosities, diffusion constants, and friction constants of these systems at $293.15 \mathrm{~K}$ and at the same densities by carrying out equilibrium molecular dynamics (EMD) simulations. The results are listed in Table 3 . It is well known that the stress auto-correlation (SAC) function for the shear viscosity, the integrand of Eq. (1), has a non-decaying long-time tail, which makes it difficult to calculate the shear viscosity. In contrast with the SAC function, the velocity auto-correlation (VAC) function, the integrand of the corresponding Green-Kubo formula for the calculation of self-diffusion coefficient, decays quickly. The difference between two auto-correlation functions is that the $\mathrm{VAC}$ deals with the velocities of the particles, while the SAC deals with the pressure tensor of the system. The VAC function can be averaged by the number of particles in the system which improves the statistical accuracy, but the SAC cannot since the pressure is the property of the system. An alternative to overcome this problem for the $\mathrm{SAC}$ is recently proposed ${ }^{16}$ which considers the pressure as a property of each particle $i$. The application of this method to a simple molecular system such as liquid argon shows a good agreement with the experimental result for viscosity. ${ }^{15}$ But the viscosity results for liquid benzen, toluene, and p-xylene underestimate the experimental result. Other EMD simulation study reported rather accurate results for the viscosity of liquid benzene and toluene. ${ }^{17}$

Diffusion constants are calculated from the mean square displacement (MSD) using the Einstein formula ${ }^{18}$ :

$$
D-\frac{1}{6} \lim _{i, m} \frac{d}{d t}\left\langle\left|\boldsymbol{r}_{i}(t)-\boldsymbol{r}_{i}(0)\right|\right\rangle^{2}
$$

and friction constants are obtained from the time integral of the force auto-correlation function (FAC) $)^{19,2 n}$ :

$$
\zeta-\frac{1}{3 k T} \int_{i j}^{T} d t\left\langle f_{i}(t) \cdot f_{i}(0)\right\rangle
$$

Table 3. The viscosity ( $n$ in $\mathrm{cP}$ ). diflusion constant ( $\mathrm{D}$ in $10^{-5} \mathrm{~cm}^{2} / \mathrm{s}$ ). and friction constant $(\zeta$ in $\mathrm{kg} / \mathrm{m}$ ) $\mathrm{ps}$ ) of the liquid benzene. toluene. and $p$-xylene at $293.15 \mathrm{~K}$ by equilibrium molecular dy namics (l:MI) simulations

\begin{tabular}{ccc|ccc|ccc}
\hline \multicolumn{3}{c|}{} & \multicolumn{3}{c|}{ toluene $(0.933)^{\prime \prime}$} & \multicolumn{3}{c}{$p$-xylene $(0.953)^{s}$} \\
\hline$n$ & $\mathrm{D}$ & $\zeta$ & $\eta$ & $\mathrm{D}$ & $\zeta$ & $\eta$ & $\mathrm{D}$ & $\zeta$ \\
\hline 0.245 & 1.94 & $0.510(1.26)^{h}$ & 0.224 & 1.85 & $0.556(1.32)^{h}$ & 0.197 & 1.80 & $0.619(1.35)^{h \prime}$ \\
\hline
\end{tabular}

"Density $\left(g / \mathrm{cm}^{3}\right)$ of each simulated system. "The values in parenthesis are friction constants oblained by Eq. (16). 
where $f_{i}(t)=\boldsymbol{F}_{i}(t)-\left\langle\boldsymbol{F}_{i}(t)\right\rangle$ and $\boldsymbol{F}_{j}(t)$ is the total force exerted on molecule $i$. We could obtain the friction constants by the time integral of the total FAC with choosing the upper linit of $\tau$ as the time which the FAC has the first negative value by assuming that the fast random force correlation ends at that time. This friction constant is related to the diffusion constant:

$$
\zeta=k T / D \text {. }
$$

Table 3 contains the friction constants obtained from the time integral of the FAC using Eq. (15) and from Eq. (16) with D obtained from MSD's in Table 3. Both the friction constants give a correct qualitative trends: decrease with increasing temperature and increase with increasing chain length.

Acknowledgment. This research was supported by Kyungsung University Special Research Grants in 2003.

\section{References}

1. Green. M. S. J. Chem. Phvs. 1951. 19. 249. ibid. 1952. 20. 281 : ibid. 1954. 22. 398: Kubo. R. J. Plys. Soc. Japam 1957. 12. 570.
2. Evans, D. J.; Morris, G. P. Comput. Plns. Rep. 1984, 1. 297

3. Evans. D. J.: Morris. G. P. Plys. Rev A 1984. 30. 1528.

4. Ciccotti. G.: Tacucci. G.: McDonald. I. R. J. Stat. Phns. 1979. 21.

5. Morriss. G. P. Evans. D. J. Hol Pho $1985,54,135$.

6. Lees. A. W.: Edwards. S. F. J. Phys, 1972, C5, 1921.

7. Evans, D. J. Hol. Ploss. 1977. 34. 317.

8. Evans. D. J.: Murad. S. M fol. Phys. 1977.34. 327.

9. Yamada. T.: Kawasaki. K. Prog. Theot Phws 1975.53. 111.

10. Kawasaki. K.: Gunton. T. D. Phs. Rev 1973. 48. 2048.

11. Ernst. M. H.: Cichocki. B; Dorfman, J. R:; Shamna. J.: van Beijeren, H. J. Stat. Phus. 1978, 18, 237.

12. Kim. J. H.: Lee, S. H. Bull. Konan Chem Soc. 2002. 23. 411.

13. Kim. J. H.: Lee. S. H. Bull. Kowan Chem Soc. 2002. 23. 447.

14. Gear. C. W. Numerical Intial Ialte Problents in Ordinary Differential Equation: Prentice-Hall: Englewood Cliffs. N.I. 1971 .

15. Landolt-B mstein, Numerical Data and Functional Relationships in Science and Techology, $6^{\text {th }}$ Ed: 1969; Vol. II $5 \mathrm{a}$.

16. Lee. S. H.: Park. D. K.: Kang. D. B. Bull. Koram Chem. So. 2003. 2+. 178

17. Kim1. J. R.: Won1. Y. Bull. Korean Chem. Soc. 2001. 22. 1287.

18. MeQuarrie. D. A. Statistical Mechanics; Harper and Row: New York. 1976.

19. Ciecotti, G; Ferrario. M; Hynes, J. T.: Kapral, R. J. Chem Plys. 1990. 93.7137.

20. Kubo. R. Rep. Prog. Phys. 1966. 29.255. 